\title{
Práticas e mecanismos de compartilhamento de conhecimento em um programa de aceleração de startups
}

\author{
Mônica Ramos Carneiro Doutoranda, Universidade Federal de Santa Catarina (UFSC) Brasil - moni.carneiro@hotmail.com \\ Thiago Furlani Zilinksi Mestrando, Universidade Federal de Santa Catarina (UFSC) Brasil - furlanidn@gmail.com \\ Eduardo Moreira da Costa Doutor, Universidade Federal de Santa Catarina (UFSC) Brasil - educostainovacao@gmail.com
}

\begin{abstract}
Resumo
Programas de aceleração de startups realizados por empresas surgiram com a intenção de aliar o potencial inovador das startups com os recursos existentes nas corporações. Sabe-se que essa união de conhecimentos, recursos e competências possibilita inovações conjuntas que trazem benefícios para ambos. Contudo, para que este tipo de aceleração ocorra, o compartilhamento do conhecimento e a criação conjunta de novos conhecimentos são de grande importância. Durante a construção dos programas de aceleração, as práticas e processos para incentivar a troca de conhecimentos devem ser bem planejados. Nesse sentido, esse artigo apresenta o estudo de caso realizado em um programa de aceleração realizado por uma empresa de base tecnológica, e que teve como objetivo identificar as práticas e mecanismos de compartilhamento de conhecimento utilizados nesse programa. A partir de uma revisão da literatura sobre o tema e do caso estudado, pode-se concluir que a aceleração de startups apoiada por uma corporação potencializa o relacionamento interpessoal entre os integrantes do programa, bem como fortalece a cultura inovadora. Esse comportamento favorece o compartilhamento de conhecimento entre os participantes, trazendo benefícios na alavancagem dos negócios para ambas as partes.
\end{abstract}

Palaras chave: gestão do conhecimento; aceleração; startups; compartilhamento de conhecimento.

\section{Practices and knowledge sharing mechanisms in a program of acceleration of startups}

\begin{abstract}
Startups acceleration programs supported by companies began with the intention of combining the innovative potential of startups with existing resources in corporations. It is known that this combination of knowledge, resources and expertise enables joint innovations that benefit them both. However, for this kind of acceleration to occur the sharing of knowledge and the joint creation of new knowledge is of great importance. Therefore, during construction of acceleration programs, practices and processes to encourage the exchange of knowledge should be well planned. In this sense, this article presents a case study in an acceleration program conducted by a technology-based company, aiming at identifying the practices and knowledge sharing mechanisms used in that program. From a literature review on the topic and the case study, it can be concluded that the acceleration of startups supported by a corporation enhances the interpersonal relationship between program participants and strengthens the innovative culture. This behavior promotes knowledge sharing among participants, bringing benefits in business for both.
\end{abstract}

Keywords: knowledge management; acceleration; startups; knowledge sharing. 


\section{INTRODUÇÃO}

A combinação da globalização com as tecnologias da informação tornou as mudanças cada vez mais rápidas, onde um conhecimento pode tornar-se obsoleto rapidamente, exigindo que as empresas renovem e ampliem seus recursos e capacidades produtivas constantemente (Lundvall \& Nielsen, 1999; Teece \& Pisano, 1997). Nesta chamada economia do conhecimento é atribuída destacada importância à inovação como pilar para o desenvolvimento econômico e social. Por este motivo, muitos esforços são empregados na pesquisa e no desenvolvimento de novos conhecimentos, processos, tecnologias e produtos (Volberda et al., 2013; Zhu, 2013). Não obstante, as empresas que têm intenção de obter sucesso em seus mercados devem ter a capacidade de inovar mais rápido que seus competidores, identificando novas formas de negócio, desenvolvendo novas tecnologias, produtos e entrando em novos mercados de forma mais eficiente (Teng, 2007; Zhu, 2013).

Zahra e Covin (1995:227) definem essas atitudes como empreendedorismo corporativo, ou "a soma da capacidade inovadora, de renovação, e de investimentos de riscos", onde a inovação é caracterizada pela criação e comercialização de tecnologia e produtos, a renovação envolve revitalizar o negócio da empresa voltado para melhorar a competitividade e os investimentos de risco se referem ao processo de fomentar e criar novas unidades de negócios. Nesse cenário, é importante que as corporações possuam o conhecimento necessário para inovar, administrando o conhecimento organizacional que já possui e eliminando barreiras estruturais, procedurais ou culturais que limitam a sua capacidade interna de produção de conhecimento e sua capacidade criativa.

O tamanho e complexidade das grandes corporações têm atrapalhado e diminuído a velocidade dos processos de inovação internos e o que se observa é o crescimento rápido nos últimos anos no número de empreendedores e negócios inovadores guiados por startups (Anthony, 2012). Esse contexto tem conduzido mudanças nas grandes corporações que têm se orientado para novas formas de inovar, levando à realização de mudanças para estruturas mais flexíveis, incentivando o comportamento empreendedor de seus funcionários. Nesta busca por alternativas externas para inovar, incubadoras e aceleradoras têm chamado a atenção de grandes corporações e investidores que reconhecem nas startups oportunidades para inovar ou para realizar parcerias. Estas são apenas uma pequena parte do ecossistema de inovação atual, mas têm se transformado em importante ferramenta para a criação de empresas inovadoras intensivas em tecnologia e conhecimento (Bruneel et al., 2012; Lewis, 2011; Miller \& Bound, 2011).

O interesse em startups é crescente e Kohler (2016) afirma que são elas quem estão liderando as grandes inovações e substituindo tecnologias e modelos de negócio no mercado. Aceleradoras são modelos de desenvolvimento de startups mais recentes e têm se espalhado pelo mundo nos últimos anos, saindo do eixo americano-europeu em direção a outros países. A literatura existente sobre o fenômeno das aceleradoras e programas de aceleração ainda é limitada devido a contemporaneidade desse modelo (Hochberg, 2015; Miller \& Bound, 2011).

Estes recentes programas de aceleração são então oportunidades para grandes empresas e startups unirem suas capacidades e qualidades para inovarem e irem juntas ao mercado, suprindo as deficiências existentes em cada lado. Para isso, durante estes programas são necessárias práticas que facilitem e promovam o compartilhamento de conhecimento entre os envolvidos tais como as identificadas por Freitas et al. (2012): benchmarking, coaching, comunidades de prática, documentação, mentoring, reuniões informais, reuniões formais, treinamentos, universidades corporativas e workshops.

O compartilhamento do conhecimento pode ser considerado o processo mais importante da Gestão do Conhecimento e é definido por Jian e Wang (2012) como o processo de "passagem do conhecimento pelos estágios de socialização, internalização, externalização e combinação sob os princípios e normas de cooperação, a fim de alcançar a difusão do conhecimento e a geração de novos conhecimentos". A importância do compartilhamento do conhecimento por parte das empresas nasce da necessidade de inovação, em um formato de desenvolvimento ágil e eficiente de novos serviços e produtos, pois o compartilhamento de conhecimento entre as pessoas aumenta a circulação de conhecimentos diversos, que, por sua vez, aumenta a capacidade produtiva da empresa, assim como reduz custos e a velocidade para a implementação de inovações (Jian \& Wang, 2012).

Diante desse cenário, o objetivo do estudo foi conhecer as práticas de compartilhamento de conhecimento existentes em um programa de aceleração de startups desenvolvido por uma empresa de base tecnológica situada em Florianópolis/SC/Brasil, visto a falta de estudos já mencionada. Para tanto, o artigo inicialmente apresenta uma breve revisão sobre o tema, o capítulo seguinte apresenta a metodologia utilizada para a realização da pesquisa, seguido pela a análise e discussão dos dados encontrados, onde são identificadas as práticas de gestão do conhecimento do programa de aceleração e o relatado como essas práticas são utilizadas no programa estudado. Na última seção são apresentadas as conclusões e resultados do artigo.

\section{DE INCUBADORAS À ACELERADORAS E PROGRAMAS DE ACELERAÇÃO}

\subsection{A quarta era da inovação: Startups e grandes corporações}


O trabalho de Anthony (2012) "The new corporate garage" apresenta o conceito da quarta era da inovação. Para o autor, estamos vivenciando uma nova etapa na história da inovação, fundamentada na relação entre startups e grandes corporações. A primeira era, chamada dos inventores solitários, perdurou por muitos anos da história de nossa sociedade até 1915. Neste período, inventores levavam suas ideias para as empresas, mas de forma geral eram limitadas pelas mesmas. Com a introdução das linhas de produção os custos para o desenvolvimento de novas inovações aumentaram, transferindo dos indivíduos para as empresas a maior parte da capacidade inovadora. Assim a segunda era da inovação foi marcada pelos laboratórios corporativos. A terceira era emergiu alicerçada pelo crescente mercado do capital de risco. As inovações começaram a ocorrer novamente fora das grandes empresas, entretanto esses novos empreendimentos necessitavam de formas de investimento para o seu desenvolvimento, o que ocorreu através do capital de risco.

Com o passar dos anos, inovar dentro das grandes empresas ficou cada vez mais difícil. A elevada burocracia, a competição das empresas nascentes e a velocidade com que o mercado exigia mudanças elevaram os custos e diminuíram a capacidade de inovação e a velocidade de reação das grandes corporações. A mudança que diferencia a quarta era das anteriores é a de que o tamanho, estrutura e alcance das grandes corporações que anteriormente criavam dificuldades, agora estão sendo utilizadas em conjunto com novos empreendedores para desenvolver as soluções e inovações de uma forma mais ágil (Anthony, 2012).

Grandes corporações e startups são empresas completamente diferentes, mas podem ser complementares, pois em muitos casos a carência de uma é o ponto forte da outra. Assim, essa natureza complementar indica que ambos podem se beneficiar da colaboração, onde startups teriam ganhos de execução e desenvolvimento de seus negócios e grandes corporações teriam suporte na busca por inovações. É a combinação da atividade empreendedora com a capacidade corporativa (Kohler, 2016; Weiblen \& Chesbrough, 2015).

\subsection{Modelos de incubação: uma análise histórica}

O conceito de incubadora é destinado às organizações que fornecem espaços compartilhados, serviços e suporte para empresas em fase inicial e que objetivam proporcionar aos seus incubados um sistema estratégico de agregação de valor e monitoramento de negócios (Bergek \& Norrman, 2008; Hackett \& Dilts, 2004). Os primeiros programas de incubação surgiram na década de 50 nos Estados Unidos. O primeiro modelo de incubadoras durou até a década de 80 e as incubadoras da época tinham como objetivos reestruturar economicamente as empresas participantes e criar novos empregos e desenvolvimento regional. Para tanto, proviam estrutura básica e serviços compartilhados às empresas (Mian et al., 2016).

Já a segunda geração de programas de incubação começou a oferecer uma série de serviços mais completos como por exemplo: consultorias, networking e treinamentos. Estes modelos rapidamente se difundiram como forma de resolver a falta de expertise na área de negócios que muitas empresas apresentavam (Bruneel et al., 2012; Mian et al., 2016). Por fim, nos anos 90 uma nova geração de incubadoras emergiu. Baseados no advento da internet e no surgimento de empresas voltadas para novas tecnologias, os modelos de incubação dessa geração focaram tipicamente em empresas de base tecnológica como biotecnologia, tecnologias da informação e comunicação ou tecnologias ambientais (Aernoudt, 2004; Bruneel et al., 2012; Mian et al., 2016). Foi nesta última geração de modelos de incubação que emergiu o conceito de aceleradora, que apresentava objetivos e características diferentes das gerações anteriores.

\subsection{O fenômeno das aceleradoras e programas de aceleração}

A economia digital e globalizada proporcionou o aparecimento de uma nova forma de incubação, a aceleradora como foi apresentado. Esse processo de aceleração tem se difundido nos últimos anos por todo o mundo sendo adotado principalmente por grupos privados e grandes corporações. A primeira aceleradora implementada foi a $Y$ Combinator fundada por Paul Graham em 2005, em Cambridge, Massachusetts (EUA) e que logo foi transferida para o Vale do Silício (Cohen, 2013; Cohen \& Hochberg, 2014; Pauwels et al., 2015). Ela foi inspiração para muitas outras aceleradoras que, desde então, têm se espalhado por todo o mundo devido principalmente ao fato da queda dos custos para investir em uma startup de software (Kerr et al, 2014; Hochberg, 2015). Entretanto, devido ao rápido crescimento do modelo, é muito cedo para afirmar e medir o impacto geral das aceleradoras (Miller \& Bound, 2011).

Estimou-se que em 2013 existiam aproximadamente 210 aceleradoras em todo o mundo (Mian et al., 2016; Nesta, 2015), já em pesquisa apresentada por Hochberg (2015) a estimativa na época subia para mais de 3.000 aceleradoras existentes. Identificou-se também, além do rápido crescimento na quantidade de programas, uma mudança no foco das aceleradoras que inicialmente eram mais generalistas aceitando startups e empreendedores que apresentavam negócios direcionados a diferentes áreas e que hoje trabalham com programas de focados nessas áreas (Cohen \& Hochberg, 2014).

Hochberg (2015) afirmou em seu estudo que a definição do termo aceleradora permanece discordante na literatura, onde alguns grupos de autores como Cohen \& Hochberg (2014) defendem uma definição uniforme, ao mesmo tempo que outros ainda conectam aceleradoras ao conceito de incubação. Para efeito deste artigo, aceleradoras ou programas de aceleração serão considerados os que combinam serviços distintos (difíceis e caros para um pequeno 
empresário obter) e investidores, resultando numa provável redução dos custos para os empreendedores e um aumento no potencial de alavancagem das startups via investimentos de capital de risco (Hochberg, 2015; Mian et al., 2016).

\section{PRÁTICAS E PROCESSOS DE COMPARTILHAMENTO DE CONHECIMENTO}

A Gestão do Conhecimento tem papel fundamental para a execução de programas de inovação que requerem colaboração, como a aceleração de startups, pois através dela pode se estruturar os fluxos de conhecimento e assegurar que ocorram as etapas, processos e práticas que proporcionam a troca e criação dos conhecimentos necessários para a construção das inovações. Por esses motivos é que a gestão do conhecimento tem se tornado uma das abordagens mais utilizadas para melhorar o desempenho das organizações (Clarke \& Cooper, 2000).

É consenso para os pesquisadores em gestão do conhecimento (Nonaka \& Takeuchi, 2008; Darroch, 2005; Crossan \& Apaydin, 2010), que o conhecimento organizacional atualmente é o recurso mais importante nas organizações, podendo se tornar uma vantagem competitiva, mas que não pode ser facilmente replicado. Assim, tanto para grandes, como para pequenas organizações e startups, a Gestão do Conhecimento (GC) ganhou notoriedade tendo como principais aspectos a gestão da informação ou conhecimento e a gestão das pessoas, ambos com o intuito de tornar o conhecimento gerado na interna ou externamente mais produtivo, bem como trazer benefícios e proporcionar a inovação dentro da organização.

Para esse estudo, a GC pode ser definida como a ferramenta para a organização gerenciar, armazenar e criar novos conhecimentos, os disseminando por toda a organização e os incorporando em produtos, serviços e sistemas gerando assim valor para a organização (Nonaka \& Takeuchi, 1995; Gupta, 2000; Liebowitz \& Wilcox, 1997). Considera-se também que o objetivo principal da GC é fomentar a estratégia de criação, transferência e aplicação do conhecimento organizacional (Jian \& Wang, 2012). Entretanto, ela por si só não promove a colaboração dentro das organizações, sendo uma abordagem colaborativa que necessita de um contexto que instigue o compartilhamento entre os diferentes atores (Clarke \& Cooper, 2000).

\section{PROGRAMA DE ACELERAÇÃO: O CASO ESTUDADO}

O caso escolhido para esse estudo é um programa de aceleração criado por uma empresa que atua no ramo tecnológico e é referência nacional no desenvolvimento de softwares de gestão, sendo ofertados desde softwares de gestão de pessoas e segurança até ERP. A empresa destaca a inovação como um de seus principais valores e, em março de 2015, lançou um programa de aceleração corporativa em Florianópolis/SC, com o objetivo de atrair startups inovadoras e inovar junto com elas.

O programa de aceleração estudado teve duração de nove meses e participaram dez startups de diferentes regiões do Brasil. O programa contou com participação ativa da empresa patrocinadora, aliada a uma aceleradora que auxiliou na organização e no planejamento do processo. O programa contou com quatro fases distintas: 1) Processo Seletivo, 2) Apresentação do Processo de Aceleração, 3) Aceleração e Mentoria e 4) Acesso ao Mercado. Em cada uma dessas fases foram utilizadas diferentes práticas de gestão do conhecimento, em diferentes níveis e com diferentes ações. As fases do programa de aceleração e Práticas de Gestão do Conhecimento utilizadas são apresentadas na seção seguinte.

\subsection{Fase 1 - Processo Seletivo}

O objetivo do processo seletivo foi escolher um portfólio de empresas diversificado, com startups em diferentes setores e tecnologias, com perfil de investimento balanceado entre conservadores e arrojados, para escolher assim as melhores startups para participar do programa. O processo seletivo foi dividido em quatro fases: a) Divulgação do programa de aceleração e inscrições, b) Seleção das startups por aderência às áreas de atuação da empresa promotora do programa de aceleração, c) Pitch e d) Banca. No decorrer das fases do processo seletivo foram utilizadas as práticas de GC: repositórios de conhecimento, comunidades de prática e mentoring.

\subsubsection{Repositório de conhecimento.}

As inscrições feitas pelos participantes foram analisadas para escolher as startups com maior aderência e capacidade empreendedora. Para isso, ocorreram entrevistas por telefone e via Skype com aquelas que apresentaram propostas mais atraentes. Após a primeira seleção, foi elaborado um dossiê das vinte startups melhor qualificadas, que foi disponibilizado para os participantes do processo e também para a banca final, composta por membros do conselho e direção da empresa promotora do programa de aceleração. Esse documento foi disponibilizado em uma pasta na nuvem para que a equipe da equipe avaliadora tivesse acesso. 


\begin{tabular}{|c|c|c|c|c|}
\hline Prática & Ação & Nível & Ferramenta & Descrição \\
\hline $\begin{array}{l}\text { Repositório de } \\
\text { conhecimento }\end{array}$ & $\begin{array}{c}\text { Aquisição e } \\
\text { compartilhamento de } \\
\text { conhecimento }\end{array}$ & Equipe & Dropbox ${ }^{1}$ & $\begin{array}{c}\text { Armazenamento de documentos } \\
\text { do processo seletivo em pasta } \\
\text { compartilhada }\end{array}$ \\
\hline
\end{tabular}

\section{Tabela 1 - Repositório de conhecimento}

Fonte: Elaborada pelos autores.

\subsubsection{Mentoring.}

As startups selecionadas na primeira fase passaram por um treinamento para o Pitch (rápida apresentação de 7 minutos). Na preparação para o Pitch houve o mentoring, onde o empreendedor foi treinado para apresentar a startup, seu modelo de negócio, modelo de monetização, mercado e equipe.

\begin{tabular}{|c|c|c|c|c|}
\hline Prática & Ação & Nível & Ferramenta & Descrição \\
\hline Mentoring & $\begin{array}{c}\text { Aquisição e } \\
\text { compartilhamento } \\
\text { de conhecimento e } \\
\text { desenvolvimento de } \\
\text { competências }\end{array}$ & $\begin{array}{c}\text { Equipe e } \\
\text { individual }\end{array}$ & $\begin{array}{c}\text { Armazenamento de } \\
\text { documentos do processo } \\
\text { seletivo em pasta } \\
\text { compartilhada }\end{array}$ \\
\hline
\end{tabular}

Tabela 2 - Mentoring

Fonte: Elaborada pelos autores.

\subsubsection{Comunidades de prática.}

Depois de receberem as instruções sobre como realizar o Pitch, as startups fizeram sua apresentação para as demais participantes, que deveriam compartilhar as críticas e sugestões sobre a apresentação. Após a primeira apresentação, a startup poderia aprimorar sua performance e apresentar novamente para as demais, seguindo as orientações e melhorias propostas. O controle do tempo foi fundamental nessa etapa, bem como a apresentação do modelo de negócio que deveria ficar claro para a banca final.

\begin{tabular}{|c|c|c|c|c|}
\hline Prática & Ação & Nível & Ferramenta & Descrição \\
\hline $\begin{array}{c}\text { Comunidade de } \\
\text { Prática }\end{array}$ & $\begin{array}{c}\text { Colaboração e } \\
\text { compartilhamento } \\
\text { de conhecimentos; } \\
\text { desenvolvimento de } \\
\text { competências }\end{array}$ & $\begin{array}{c}\text { Equipe e } \\
\text { individual }\end{array}$ & $\begin{array}{c}\text { Armazenamento de } \\
\text { documentos do processo } \\
\text { seletivo em pasta } \\
\text { compartilhada }\end{array}$ \\
\hline
\end{tabular}

\section{Tabela 3 - Comunidades de Prática}

Fonte: Elaborada pelos autores.

\subsection{Fase 2 - Apresentação do Processo de Aceleração}

Os ciclos de aceleração foram de uma semana de imersão com a participação das startups selecionadas para participar do programa de aceleração. O primeiro ciclo teve seu foco na apresentação do programa para as startups e das startups para a aceleradora e a empresa patrocinadora, de uma forma mais detalhada e completa. Esse ciclo aconteceu

1 Drive de armazenamento na nuvem. 
em torno da prática de gestão do conhecimento de comunidades de prática. As startups foram divididas em três grupos de acordo com o seu nível de maturidade. Assim, no nível 1 ficaram as startups que ainda não tinham Produto Mínimo Viável (MVP) validado ou que precisavam amadurecer o escopo da solução ou o modelo de negócio, no nível 2 ficaram as startups que já tinham um MVP no mercado ou um protótipo funcional ou mesmo uma versão beta e no nível 3 ficaram as startups que já possuíam uma oferta madura e clientes, mas precisavam validar hipóteses, avaliar sua capacidade e se prepararem melhor para o mercado.

\subsubsection{Comunidades de prática.}

Durante a semana de imersão aconteceram comunidades de prática onde os participantes do nível 2 apresentavam sua startup na parte da manhã para um diagnóstico de: apresentação do produto; equipe; do estágio de desenvolvimento; dos clientes; análise SWOT; apresentação, elaboração e revisão de um (lean) Canvas. Nas tardes, o objetivo era a cocriação do percurso individual de aceleração: premissas/evidências; validação da solução/modelo de negócio; métricas de acompanhamento. Nessa dinâmica, uma startup por dia fazia a apresentação enquanto era assistida e recebia críticas comentários e sugestões dos demais.

\begin{tabular}{|c|c|c|c|c|}
\hline Prática & Ação & Nível & Ferramenta & Descrição \\
\hline & $\begin{array}{c}\text { Colaboração e } \\
\text { compartilhamento de } \\
\text { conhecimentos; }\end{array}$ & $\begin{array}{c}\text { Armazenamento de } \\
\text { Comunidade de } \\
\text { Prática }\end{array}$ & $\begin{array}{c}\text { Aquisição de } \\
\text { conhecimento; } \\
\text { individual } \\
\text { desenvolvimento de } \\
\text { competências. }\end{array}$ & $\begin{array}{c}\text { Dropbox e Trello }{ }^{2} \\
\text { seletivo em pasta } \\
\text { compartilhada }\end{array}$ \\
\hline
\end{tabular}

Tabela 4 - Comunidades de Prática

Fonte: Elaborada pelos autores.

\subsection{Fase 3 - Aceleração e Mentoria}

O Segundo Ciclo de imersão durou uma semana e teve como foco as áreas de Tecnologias (arquitetura); Produto (recursos; proposta de valor; MVP) e Mercado (discurso de venda; preço; acesso a clientes). No entanto, nesse ciclo a principal prática de gestão do conhecimento não foram as comunidades de prática, mas sim as redes de especialistas. As startups se apresentaram, em formato de Pitch, a uma equipe de especialista da patrocinadora, chamados anjos corporativos, os quais também estiveram em reuniões individuais de trabalho de acordo com as necessidades e momento das startups. A equipe de anjos foi composta por 16 especialistas nas áreas de: ERP, técnico de sistemas, gestão de produto, gerência de produto, performance corporativa, marketing, pesquisa e arquitetura, desenvolvimento, jurídico, inteligência competitiva, gestão de pessoas, consultoria e implantação de serviços, comercial e pré-vendas, processo de vendas, relacionamento com clientes e desenvolvimento humano na organização.

A semana começou com o alinhamento das atividades e com uma palestra sobre plataforma de produtos, na segunda-feira. Na terça-feira e quinta-feira, manhã e tarde, foram realizadas as reuniões entre startups e anjos corporativos. Na quarta-feira foi opcional para as startups agendarem visitas à sede da patrocinadora ou conversas com a equipe do programa de aceleração, em Florianópolis/SC.

\subsubsection{Mentoring.}

Com base na agenda e desafios identificados e estabelecidos nas comunidades de prática do primeiro ciclo foram definidas as agendas de encontros com especialistas de cada startup. Assim, todas as startups e anjos corporativos tiveram dois dias de reuniões - terça-feira e quinta-feira, manhã e tarde.

2 Aplicativo de gerenciamento de dashboards editáveis. 


\begin{tabular}{|c|c|c|c|c|}
\hline Prática & Ação & Nível & Ferramenta & Descrição \\
\hline $\begin{array}{c}\text { Mentoring com } \\
\text { as redes de } \\
\text { especialistas }\end{array}$ & $\begin{array}{c}\text { Aquisição, } \\
\text { colaboração e } \\
\text { de conhecimento; } \\
\text { desenvolvimento de } \\
\text { competências }\end{array}$ & $\begin{array}{c}\text { Individual e } \\
\text { intraorganizacional }\end{array}$ & Trello & $\begin{array}{c}\text { Compartilhamento dos } \\
\text { feedbacks das reuniões com } \\
\text { especialistas }\end{array}$ \\
\hline
\end{tabular}

\section{Tabela 5 - Mentoring com as Redes de Especialistas}

Fonte: Elaborada pelos autores.

\subsubsection{Cafés do conhecimento.}

Ao final dos dois dias de reunião aconteceram cafés do conhecimento. Nesse momento, buscou-se proporcionar um encontro mais descontraído entre os anjos, as startups e a equipe da patrocinadora, durante um café oferecido na copa. Dessa forma, foi possível todas as startups e todos os anjos se conhecerem e conversarem, ainda que de forma breve, para iniciar um relacionamento.

\begin{tabular}{|c|c|c|c|c|}
\hline Prática & Ação & Nível & Ferramenta & Descrição \\
\hline $\begin{array}{c}\text { Cafés do } \\
\text { conhecimento }\end{array}$ & $\begin{array}{c}\text { Colaboração e } \\
\text { compartilhamento } \\
\text { de conhecimento }\end{array}$ & Equipe & $\begin{array}{c}\text { Encontro informal entre as } \\
\text { startups, os anjos corporativos } \\
\text { e a equipe da patrocinadora }\end{array}$ \\
\hline
\end{tabular}

\section{Tabela 6 - Café do Conhecimento}

Fonte: Elaborada pelos autores.

\subsubsection{Comunidades de prática.}

Ao final do Segundo Ciclo, sexta-feira pela manhã, houve uma comunidade de prática, de forma diferenciada das que aconteceram no primeiro ciclo. Dessa vez todas as empresas estavam reunidas para dar e receber um feedback da semana de aceleração, cada uma teve a oportunidade de compartilhar de forma breve seu relato de experiência e receber as instruções para as atividades que deveriam executar até o próximo ciclo de aceleração.

\begin{tabular}{|c|c|c|c|c|}
\hline Prática & Ação & Nível & Ferramenta & Descrição \\
\hline $\begin{array}{c}\text { Comunidade de } \\
\text { Prática }\end{array}$ & $\begin{array}{c}\text { Colaboração e } \\
\text { compartilhamento } \\
\text { de conhecimentos }\end{array}$ & $\begin{array}{c}\text { Equipe e } \\
\text { Individual }\end{array}$ & $\begin{array}{c}\text { Armazenamento de } \\
\text { documentos e }\end{array}$ \\
$\begin{array}{c}\text { Drompox e Trello } \\
\text { informações do ciclo e }\end{array}$ & $\begin{array}{c}\text { atividades a serem realizadas. } \\
\text { atidamento das }\end{array}$ \\
\hline
\end{tabular}

\section{Tabela 7 - Comunidades de Prática}

Fonte: Elaborada pelos autores.

\subsection{Fase 4 - Acesso ao Mercado}

O Terceiro Ciclo do programa foi de aproximação com os conselheiros da patrocinadora. A reunião do conselho aconteceu em Florianópolis/SC, no primeiro dia do ciclo. Nesse dia, os conselheiros também se dedicaram a assistir o pitch das startups participantes do programa, uma vez que nem todos os conselheiros estiveram presentes na banca do processo seletivo. Todas as manhãs dos dias seguintes foram dedicadas a workshops cujos temas foram: workshop sobre modelos de receita e precificação; workshop sobre relacionamento e remuneração da força de venda; como encantar um $\mathrm{ClO} /$ gerente de $\mathrm{Tl} ;$ Inbound Marketing. 
A temática principal desse ciclo foi o acesso ao mercado, uma vez que no Segundo Ciclo estiveram presentes os anjos corporativos, com dez especialistas da patrocinadora e trabalharam as temáticas: modelo de receita; precificação; canais; remuneração de canais; preparar para comercialização pioneira: validar/criar diferenciais, validar discurso/oferta; Universidade corporativa, treinamento e relacionamento com força de vendas. Logo, as Práticas de gestão do conhecimento do Terceiro Ciclo estavam concentradas em mentoring com a rede de especialistas, café do conhecimento e comunidades de prática.

\subsubsection{Mentoring.}

Os anjos corporativos tiveram agendas de reuniões como as do Segundo Ciclo, individuais com cada startup, de acordo com a necessidade de cada uma e a especialidade do anjo.

\begin{tabular}{|c|c|c|c|c|}
\hline Prática & Ação & Nível & Ferramenta & Descrição \\
\hline $\begin{array}{c}\text { Mentoring com } \\
\text { as redes de } \\
\text { especialistas }\end{array}$ & $\begin{array}{c}\text { Aquisição, } \\
\text { colaboração e } \\
\text { compartilhamento } \\
\text { de conhecimento; } \\
\text { desenvolvimento de } \\
\text { competências }\end{array}$ & $\begin{array}{c}\text { Individual e } \\
\text { intraorganizacional }\end{array}$ & Trello & $\begin{array}{c}\text { Compartilhamento dos } \\
\text { feedbacks das reuniões com } \\
\text { especialistas }\end{array}$ \\
\hline
\end{tabular}

\section{Tabela 8 - Mentoring com as Redes de Especialistas}

Fonte: Elaborada pelos autores.

\subsubsection{Café do conhecimento.}

Ao final do primeiro dia houve um Café do Conhecimento, para que startups e conselheiros pudessem se conhecer.

\begin{tabular}{|c|c|c|c|c|}
\hline Prática & Ação & Nível & Ferramenta & Descrição \\
\hline $\begin{array}{c}\text { Cafés do } \\
\text { conhecimento }\end{array}$ & $\begin{array}{c}\text { Colaboração e } \\
\text { compartilhamento } \\
\text { de conhecimento }\end{array}$ & Equipe & $\begin{array}{c}\text { Encontro informal entre as } \\
\text { startups, os anjos corporativos } \\
\text { e a equipe da patrocinadora. }\end{array}$ \\
\hline
\end{tabular}

\section{Tabela 9 - Café do Conhecimento}

Fonte: Elaborada pelos autores.

\subsubsection{Comunidades de prática.}

Ao final do Terceiro Ciclo, houve uma comunidade de prática na qual, como no Segundo Ciclo, todas as empresas estavam reunidas com a equipe da patrocinadora para fazer o alinhamento e fechamento da semana e compartilhar o feedback e instruções para o próximo ciclo.

\begin{tabular}{|c|c|c|c|c|}
\hline Prática & Ação & Nível & Ferramenta & Descrição \\
\hline $\begin{array}{c}\text { Comunidade de } \\
\text { Prática }\end{array}$ & $\begin{array}{c}\text { Colaboração e } \\
\text { compartilhamento } \\
\text { de conhecimentos }\end{array}$ & $\begin{array}{c}\text { Equipe e } \\
\text { Individual }\end{array}$ & $\begin{array}{c}\text { Armazenamento de } \\
\text { documentos e }\end{array}$ \\
Dropbox e Trello \\
compartilhamento das \\
informações do ciclo e \\
atividades a serem realizadas.
\end{tabular}

\section{Tabela 10 - Comunidades de Prática}

Fonte: Elaborada pelos autores. 
A tabela a seguir resume as práticas de gestão do conhecimento, o nível onde acontece e as ações de gestão, que foram base para as análises feitas na apresentação dos resultados do presente estudo.

\begin{tabular}{|c|c|c|}
\hline NÍVEL & $\begin{array}{l}\text { PRÁTICAS DE GESTÃO DO } \\
\text { CONHECIMENTO }\end{array}$ & $\begin{array}{l}\text { AÇÕES DE GESTÃO DO } \\
\text { CONHECIMENTO }\end{array}$ \\
\hline Individual (startup) & $\begin{array}{l}\text { Mentoring } \\
\text { Coaching } \\
\text { Repositórios de conhecimento } \\
\text { Ambientes virtuais para conversa }\end{array}$ & $\begin{array}{l}\text { Desenvolvimento de competências } \\
\text { Aquisição de conhecimentos }\end{array}$ \\
\hline $\begin{array}{c}\text { Equipe (Startup + Aceleradora + } \\
\text { Patrocinadora) }\end{array}$ & $\begin{array}{l}\text { Comunidades de prática } \\
\text { Espaço de Coworking } \\
\text { Café do conhecimento }\end{array}$ & $\begin{array}{l}\text { Colaboração e compartilhamento de } \\
\text { conhecimentos }\end{array}$ \\
\hline Intraorganizacional & $\begin{array}{l}\text { Redes de especialistas (anjos } \\
\text { corporativos) }\end{array}$ & $\begin{array}{l}\text { Colaboração e compartilhamento de } \\
\text { conhecimentos }\end{array}$ \\
\hline Interorganizacional & $\begin{array}{l}\text { Benchmarking externo } \\
\text { Contato direto com clientes }\end{array}$ & Validação de conhecimentos \\
\hline
\end{tabular}

\section{Tabela 11 - Práticas de GC}

Fonte: Elaborado pelos autores.

Como se pode perceber, as ações de gestão do conhecimento adotadas no programa de aceleração envolviam o desenvolvimento de competências, aquisição de conhecimentos, colaboração, compartilhamento e validação de conhecimentos.

\section{CONSIDERAÇÕES FINAIS}

Programas de aceleração são oportunidades para grandes empresas e startups unirem suas capacidades, experiências e qualidades para inovar e irem juntas ao mercado, suprindo as deficiências existentes em cada lado. Dessa forma, esses programas estão se tornando a solução encontrada por várias organizações para alcançar um potencial inovador, o desenvolvimento e a aquisição de conhecimento, de uma forma que não seria possível em sua estrutura organizacional interna.

As práticas de gestão do conhecimento são muito importantes nesse momento para que a organização e as startups possam compartilhar, avaliar e monitorar seu desempenho e para que não se perca o conhecimento que é trocado nesse ambiente compartilhado. Como resultados da utilização das práticas de gestão do conhecimento no programa de aceleração percebeu-se que as comunidades de prática deram a cada uma das startups uma nova visão sobre seus negócios e sobre o caminho que iriam percorrer durante o programa.

As práticas foram analisadas de acordo com seu uso durante as fases do programa de aceleração. Foram identificadas as práticas: repositórios de conhecimento, comunidades de prática e mentoring, café do conhecimento. Então em cada momento foi feita a análise da ação, ou seja, o que estava acontecendo especificamente naquela prática naquele momento. Em uma comunidade de prática, por exemplo, houve em diferentes momentos colaboração, compartilhamento de conhecimento e desenvolvimento de competências. Feita essa análise foi identificado se a prática aconteceu em nível individual ou equipe, foram registradas as ferramentas de armazenamento do conhecimento e uma breve descrição da prática realizada.

As duas práticas mais comuns no programa de aceleração foram as comunidades de prática e o mentoring, que aconteceram a cada ciclo, envolvendo os participantes a nível individual e a equipe. O ponto forte dessas ações foi a colaboração e o compartilhamento de conhecimento, o que demostrou ser, para as startups, um diferencial do programa, pois além de ter acesso aos especialistas de uma grande empresa de TI. Com essas práticas as startups também puderam compartilhar seu processo com as demais startups do programa, o que gerou um resultado muito positivo, como elas estavam em diferentes níveis de maturidade organizacional se beneficiaram da ajuda de outras mais que já haviam passado pelas mesmas situações.

O lugar onde o programa aconteceu foi um espaço de coworking, o que foi muito vantajoso pois, por estarem presentes em um mesmo espaço, as startups e a equipe de aceleração puderam conversar e resolver problemas de forma mais ágil. Adicional a isso, também tem se mostrado importante para fortalecer o relacionamento interpessoal entre os 
integrantes do programa, para que se tenha um clima organizacional bom e o compartilhamento aconteça de forma natural. Nesse sentido, pode-se concluir que a aceleração colaborativa entre startups e uma grande corporação tem importante não só para o relacionamento interpessoal entre os integrantes desse tipo de programa, bem como fortalecer uma cultura inovadora e o compartilhamento de conhecimento entre os participantes.

O espaço de coworking também tem se mostrado importante para o relacionamento interpessoal entre os integrantes do programa, para que se tenha um bom clima organizacional e o compartilhamento aconteça de forma natural. Nesse sentido, pode-se concluir que a aceleração coorporativa de startups, da forma colaborativa como aconteceu nesse programa, é vantajosa não só para nos aspectos relacionados ao negocio, mas também para o relacionamento interpessoal entre os integrantes do programa, fortalecendo uma cultura inovadora e o compartilhamento de conhecimento entre os participantes.

\section{REFERÊNCIAS}

Aernoudt, R. (2004). Incubators: tool for entrepreneurship?. Small Business Economics, 23(2), 127-135.

Anthony, S. D. (2012). The new corporate garage. Harvard Business Review, 90(9), 44-53.

Bergek, A., \& Norrman, C. (2008). Incubator best practice: A framework. Technovation, 28(1), 20-28.

Bruneel, J., Ratinho, T., Clarysse, B., \& Groen, A. (2012). The evolution of business incubators: Comparing demand and supply of business incubation services across different incubator generations. Technovation, 32(2), 110-121.

Clarke, P., \& Cooper, M. (2000, October). Knowledge management and collaboration. CiteSeer, 1-9.

Cohen, S. (2013). What do accelerators do? Insights from incubators and angels. innovations, $8(3-4), 19-25$.

Cohen, S., \& Hochberg, Y. V. (2014). Accelerating startups: The seed accelerator phenomenon. Recuperado de http://dx.doi.org/10.2139/ssrn.2418000

Crossan, M. M., \& Apaydin, M. (2010). A multi-dimensional framework of organizational innovation: A systematic review of the literature. Journal of Management Studies, 47(6), 1154-1191.

Darroch, J. (2005). Knowledge management, innovation and firm performance. Journal of Knowledge Management, 9(3), 101-115.

Freitas, R. M., Morais, P., \& Lopes, F. (2012, September). Knowledge management practices: A framework proposal. Proceedings of the European Conference on Knowledge Management, Spain.

Gupta, B., lyer, L. S., \& Aronson, J. E. (2000). Knowledge management: Practices and challenges. Industrial Management \& Data Systems, 100(1), 17-21.

Hackett, S. M., \& Dilts, D. M. (2004). A systematic review of business incubation research. The Journal of Technology Transfer, 29(1), 55-82.

Hochberg, Y. V. (2016). Accelerating entrepreneurs and ecosystems: The seed accelerator model. Innovation Policy and the Economy, 16(1), 25-51.

Jian, Z., \& Wang, C. (2012, July). The impact of relational embeddedness, knowledge sharing on service innovation performance. Proceedings of International Conference on Service Systems and Service Management, 9.

Keil, T., Maula, M., Schildt, H., \& Zahra, S. A. (2008). The effect of governance modes and relatedness of external business development activities on innovative performance. Strategic Management Journal, 29(8), 895-907.

Kerr, W. R., Nanda, R., \& Rhodes-Kropf, M. (2014). Entrepreneurship as experimentation. The Journal of Economic Perspectives, 28(3), 25-48.

Kohler, T. (2016). Corporate accelerators: Building bridges between corporations and startups. Business Horizons, 59(3), 347-357.

Labiak Junior, S. (2012). Método de análise dos fluxos de conhecimento em sistemas regionais de inovação (Tese de Doutorado, Universidade Federal de Santa Catarina). Recuperado de http://repositorio.ufsc.br/xmlui/handle/123456789/100806 
Lewis, D. A. (2001). Does technology incubation work?: A critical review. Washington, DC: Economic Development Administration, US Department of Commerce.

Liebowitz, J., \& Wilcox, L. C. (1997). Knowledge management and its integrative elements. Boca Raton, Flórida: CRC Press.

Lundvall, B. A., \& Nielsen, P. (1999). Competition and transformation in the learning economy-lllustrated by the Danish case. Revue d'économie industrielle, 88(1), 67-89.

Mian, S., Lamine, W., \& Fayolle, A. (2016). Technology business incubation: An overview of the state of knowledge. Technovation, 50, 1-12.

Miller, P., \& Bound, K. (2011). The startup factories: The rise of accelerator programs to support new technology ventures. Recuperado de https://www.nesta.org.uk/sites/default/files/the_startup_factories_0.pdf

Clarisse, B.; Wright, M., \& Van Hove, J. (2015). A look inside accelerators: Building business. Recuperado de https://www.nesta.org.uk/sites/default/files/a_look_inside_accelerators.pdf

Nonaka, I., \& Takeuchi, H. (1995). The knowledge-creating company: How Japanese companies create the dynamics of innovation. Oxford: Oxford University Press.

Pauwels, C., Clarysse, B., Wright, M., \& Van Hove, J. (2016). Understanding a new generation incubation model: The accelerator. Technovation, 50, 13-24.

Rasoto, V. I. (2006). Estrutura de referência para incubadoras pertencentes a redes de habitats de inovação tecnológica e vinculadas a instituições de ensino: estudo de caso da Reparte. (Tese de Doutorado, Universidade Federal de Santa Catarina). Recuperado de http://repositorio.ufsc.br/xmlui/handle/123456789/88905

Teece, D., \& Pisano, G. (1994). The dynamic capabilities of firms: An introduction. Industrial and Corporate Change, 3(3), 537-556.

Teng, B. S. (2007). Corporate entrepreneurship activities through strategic alliances: A resource-based approach toward competitive advantage. Journal of Management Studies, 44(1), 119-142.

Trivinos, A. N. (1987). Introdução à pesquisa em ciências sociais: A pesquisa qualitativa em educação, o positivismo, a fenomenologia, o marxismo. São Paulo: Atlas.

Volberda, H. W., Van Den Bosch, F. A., \& Heij, C. V. (2013). Management innovation: Management as fertile ground for innovation. European Management Review, 10(1), 1-15.

Weiblen, T., \& Chesbrough, H. W. (2015). Engaging with startups to enhance corporate innovation. California Management Review, 57(2), 66-90.

Yin, R. K. (2015). Estudo de caso: Planejamento e métodos. Porto Alegre: Bookman.

Zahra, S. A., \& Covin, J. G. (1995). Contextual influences on the corporate entrepreneurship-performance relationship: A longitudinal analysis. Journal of Business Venturing, 10(1), 43-58.

Zhu, M. Z. (2013). Theoretical perspective in innovation management implementation: A literature review. In Qi, Ershi, Shen, Jiang, \& Dou, Runliang (Eds.). The $19^{\text {th }}$ International Conference on Industrial Engineering and Engineering Management. New York: Springer. 